\title{
Proximal spinal muscular atrophy: current orthopedic perspective
}

\author{
This article was published in the following Dove Press journal: \\ The Application of Clinical Genetics \\ 13 November 2013 \\ Number of times this article has been viewed
}

\author{
Gerrit Haaker \\ Albert Fujak \\ Department of Orthopaedic Surgery, \\ Friedrich-Alexander-Universität \\ Erlangen-Nürnberg, Erlangen, Germany
}

\begin{abstract}
Spinal muscular atrophy (SMA) is a hereditary neuromuscular disease of lower motor neurons that is caused by a defective "survival motor neuron" (SMN) protein that is mainly associated with proximal progressive muscle weakness and atrophy. Although SMA involves a wide range of disease severity and a high mortality and morbidity rate, recent advances in multidisciplinary supportive care have enhanced quality of life and life expectancy. Active research for possible treatment options has become possible since the disease-causing gene defect was identified in 1995. Nevertheless, a causal therapy is not available at present, and therapeutic management of SMA remains challenging; the prolonged survival is increasing, especially orthopedic, respiratory and nutritive problems. This review focuses on orthopedic management of the disease, with discussion of key aspects that include scoliosis, muscular contractures, hip joint disorders, fractures, technical devices, and a comparative approach of conservative and surgical treatment. Also emphasized are associated complications including respiratory involvement, perioperative care and anesthesia, nutrition problems, and rehabilitation. The SMA disease course can be greatly improved with adequate therapy with established orthopedic procedures in a multidisciplinary therapeutic approach.
\end{abstract}

Keywords: spinal muscular atrophy, scoliosis, contractures, fractures, lung function, treatment, rehabilitation, surgery, ventilation, nutrition, perioperative management

\section{Introduction}

Spinal musclular atrophy (SMA) is a hereditary neuromuscular disease. It is usually caused by autosomal-recessive inherited mutation or deletion in the survival motor neuron (telSMN; SMN1) gene on chromosome 5q, which codes for the survival motor neuron (SMN) protein. ${ }^{1}$ The gene defect leads to degeneration of the anterior horn cells of the spinal cord and occasionally of the motor neurons of the cranial nerves $\mathrm{V}$ to XII. SMA occurs with an approximate incidence of $1: 15,000$ to $1: 20,000$, with a prevalence of 1:80 among carriers of the gene. ${ }^{2}$ Despite active research and multiple approaches for new treatment options, a causative therapy for SMA is not available at present.

Proximal SMA is characterized by muscular hypotonia, hyporeflexia, and general weakness, which are most prominent in proximal muscles with prevalence of the lower extremities. The disease is classified into four different types, with subdivisions according to the severity of the disease (see "Classification" for details). Whereas SMA type Ia has a very grave disease course in which orthopedic treatment can only marginally influence disease progression, patients with adult SMA type IV are much less affected, and conservative therapy is often sufficient to improve quality of life.
Correspondence: Albert Fujak

Department of Orthopaedic Surgery,

Friedrich-Alexander-Universität

Erlangen-Nürnberg, Rathsberger Str 57,

D-91054 Erlangen, Germany

Tel +49 913। 8223935

Fax +49 913। 8523565

Email a.fujak@t-online.de 
For orthopedic treatment, SMA type II and type IIIa are the most important subgroups because adequate and early treatment, including surgical interventions, can significantly decelerate the disease progression and improve quality of life.

Characteristic symptoms of SMA, especially types II and IIIa, include contractures of the lower extremities (with hip subluxations and dislocations), as well as contractures and hypermobile joints in the upper extremities, and, less frequently, fine tremor of the fingers. Furthermore, there is a high risk for spontaneous fractures due to osteopenia and a possible interaction between the osteoclast-stimulating factor and the SMN protein.

Scoliosis emerges in nearly $100 \%$ of nonambulatory SMA patients with a severe progression, and it remains one of the major problems for orthopedic therapy. ${ }^{3}$ Consequences include chest-cage deformities, impingement of the ribs, and pelvic tilt; the consequences of respiratory problems due to partially massive constriction of the vital capacity. Furthermore, limited respiratory capacity, anesthesia problems during perioperative care, nutrition and growth difficulties, as well as the need for special rehabilitation and integration procedures should be kept in mind, especially for hospital treatment.

In the present article, we give an overview of the main orthopedic problems affecting patients with SMA. We deal with conservative and operative treatment options for contractures, hip dysplasia, fractures, scoliosis and pelvic obliquity, and possible effects on respiratory function and quality of life of the patient. We review recently published literature and our own experiences at our neuromuscular disease center in order to develop current treatment recommendations from an orthopedic perspective.

\section{Classification}

Proximal SMA is classified into four types on the basis of the maximal physical-function development correlated with the onset and severity of disease (see Table 1). ${ }^{4,5}$
Type I SMA, named Werdnig-Hoffmann disease by the first describers of the condition, occurs in $50 \%$ of patients diagnosed with $\mathrm{SMA}^{6}$ and is the most common and most severe type of SMA. The disease course involves earlychildhood deterioration, with onset ranging from the prenatal period to the first 6 months of life. SMA type I is subdivided into type Ia (a life span of up to 2.5 years) and type Ib (a life expectancy of 2.5-20 years or longer). Patients with type I SMA never acquire the ability to sit unsupported ${ }^{7,8}$ and they have profound hypotonia, often no head control, and a typical severe respiratory pattern. In the most severe forms, prenatal onset, decreased intrauterine movement, weakness and joint contractures at birth, congenital bone fractures, and chest deformities have been observed..$^{9,10}$

Type II SMA is characterized by disease onset within the first 18 months of life and an individually wide range of survival, which is significantly reduced compared with type III SMA. Patients with this intermediate SMA type achieve unsupported sitting and, rarely, standing ability, but do not pass the usual motor milestones and never walk. The clinical course is marked by periods of apparent arrest; progressive scoliosis and contractures, however, are very common and develop early in the nonambulatory patients ${ }^{11}$ with a special need for orthopedic care. Weak chewing muscles, swallowing difficulties, and early respiratory disorders are frequently present, with a wide spectrum of severity.

Type III SMA, also called Kugelberg-Welander disease, is a milder form with disease onset from the first year to the third decade of life. The disease course is characterized by slow progression and periods of apparent arrest; standing and walking ability are often preserved until adulthood. ${ }^{11}$ This type is subdivided into type IIIa and type IIIb. Type IIIa, with disease onset before 3 years of age, has a slightly reduced life span with delayed motor development; type IIIb is characterized by onset after 3 years of age, with a mostly normal life expectancy, and normal motor development. ${ }^{11,12}$ Patients with type IIIa SMA have orthopedic problems that are comparable to those experienced by patients with type II SMA,

Table I Classification of proximal SMA

\begin{tabular}{lll}
\hline SMA type & Age of onset & Achieved function \\
\hline la (more severe) & Prenatal to 6 months & No sitting or turning \\
Ib (less severe) & Prenatal to 6 months & No sitting or turning \\
II (intermediate) & Birth to I8 months & Sitting ability \\
IIla (mild; retarded motor development) & Birth to 3 years & Walking ability \\
IIlb (mild; normal motor development) & $>3-30$ years & Walking ability \\
IV (adult) & $>30$ years & Walking ability
\end{tabular}

Note: *Possible longer life expectancy.

Abbreviation: SMA, spinal muscular atrophy. 
although generally with later onset and decreased severity of the disease, whereas patients with type IIIb SMA have only mild orthopedic disturbances.

The adult type IV SMA has a late onset within the second or third decade and normal life expectancy. The mild disease course shows only marginally affected preserved walking ability; respiratory and nutritional problems are very rare. ${ }^{4,6,11}$

\section{Contractures}

\section{Upper extremities}

Limited range of motion can be found in SMA patients at 3-5 years of age, with an age-dependent progression. ${ }^{13}$ Contractures in the upper extremities are very rarely isolated, usually occurring in combination with contractures in lower extremities. Elbow joints are prevalently affected with flexion contractures and limited supination, followed by shoulder and wrist contractures. Hypermobile joints, often hypermobile wrists, are observed as well. ${ }^{14}$ Indication for surgical treatment is only rarely given. Early physiotherapy can decrease contracture progression; adjusted medical strength training for milder SMA forms can positively influence especially hypermobile joints. ${ }^{15}$ Patients with type II or type IIIa SMA and upper-extremity contractures are commonly fitted with orthoses, which are likely to have a positive effect; however, scientific information about the effect is not available at present and remains to be proven by further investigation. Continuous physiotherapy and occupational therapy are necessary to preserve remaining capabilities in daily life and can help to decrease contracture progression and to optimize residual range of motion. ${ }^{13,16}$

\section{Lower extremities}

Contractures occur regularly and more prevalently in the lower extremities than in the upper extremities. Severe loss of range of motion to the point of contractures has been found at the early age of 2 years. ${ }^{17}$ Pronounced flexion contractures of the hip and knee joint and strongly restricted mobility of the ankle joint with talipes equinus are common. ${ }^{17}$ For patients with SMA type IIIb who are able to stand, it is important to maintain this ability - in some cases with additional orthoses and technical devices. In contrast to treatment for contractures in upper extremities, surgical treatment for lower extremity contractures can be effective especially for patients with type II or type IIIa SMA, even though its effectiveness can be limited by individual differences in strong fibrotic tendency of the muscles and by the wheelchair position of nonambulatory patients. Tenotomy or lengthening of the Musculus sartorius,
Musculus tensor fasciae latae, Musculus rectus femoris, Musculus iliopsoas, the adductors, and the knee flexor muscles can be provided to patients with severe contractures of hip and knee joints. ${ }^{17}$ For affected feet, tenotomy or transposition of the Musculus tibialis posterior and tenotomy of the Musculus flexor digitorum longus or Musculus flexor hallucis longus as well as peroneal tendons have shown a positive effect in some cases. Achillotenotomy and capsulotomy of the ankle joint can become necessary as well. ${ }^{17}$ Despite the positive effect of surgery, the indication has to be assessed very carefully, and postoperative immobilization should be kept as brief as possible because a long immobilization leads to rapid deterioration of motor ability. ${ }^{17,18}$

Physiotherapy remains important for prevention and handling of lower-limb contractures for all SMA types. Orthoses and technical devices that aid standing and provide support (see "Orthoses" section) can decelerate the progression of contractures. ${ }^{17}$

\section{Hip joint involvement}

Hip subluxations are frequent in patients with SMA, mostly in nonambulatory patients. ${ }^{19-22}$ Treatment for patients with SMA type I is usually not requested because of poor general condition; $;^{19,20}$ thus, the highest incidence for orthopedicrelevant hip subluxations and dislocations can be found in SMA type II and type III patients. Subluxation occurs in approximately $30 \%-40 \%$ of SMA type II patients and $10 \%-30 \%$ of SMA type III patients. Dislocation occurs in approximately $30 \%$ of SMA type II patients and 20\%-30\% in SMA type III patients. ${ }^{19-22}$ The weakness of hip-stabilizing muscles, the lack of physiological weight-bearing and missing axialization are the reasons for recurrent hip dysplasia, subluxation, and dislocation. ${ }^{19}$ Nonetheless, clinical problems of hip dislocations and subluxations have been only rarely reported. Some sporadic cases of hip pain have been described in patients without positioning problems or limitations in daily life activities despite recurrent hip dislocation. ${ }^{19-22}$ In contrast, operatively corrected hip joints, including hips corrected with surgical reduction and osteotomy, have a higher incidence for redislocations and only a marginally better outcome after operation. ${ }^{20}$ Given the rarity of symptoms and the high risk for redislocation, and considering the significantly higher risks associated with anesthesia and surgical procedures for these patients, operative hip joint correction has very limited indications and provides only minimal functional gain. In conclusion, conservative management is the method of choice for hip dislocation and subluxation in patients with SMA type II or type III after loss of ambulation. 


\section{Fractures}

SMA-attendant fractures are a significant complication for patients with type II or type IIIa SMA, whereas congenital fractures are described primarily for SMA type I. ${ }^{23}$ Fractures occur significantly earlier in SMA type II compared with type III. Most of the fractures appear in the upper leg region, mainly supracondylar fractures followed by lower leg and ankle fractures; the distribution of fractures in SMA type IIIa differs slightly, with a higher prevalence for fractures in upper extremities. ${ }^{24}$ This can be explained by higher mobility on the one hand and unsteadiness with a resulting risk for falls and consecutive fractures on the other hand in this group. In accordance with other neuromuscular diseases, patients with SMA have a generally increased fracture risk that is worsened with the loss of ambulation and with wheelchair use. ${ }^{25}$

For nonambulatory patients, the necessity of fracture reduction depends on the type of fracture, the level of contractures, and preserved mobility. Osteosynthesis is rarely indicated for these patients, since the bones are not suitable for this procedure due to fragility, demineralization, and small size. ${ }^{26,27}$ Because poor bone quality does not allow early remobilization and functional treatment, immobilization after surgery is usually required. The demineralization and osteopenia, mainly caused by inactivity, may be deteriorated by a possible interaction between the osteoclast-stimulating factor and the SMN protein. ${ }^{28}$

Conservative treatment is the primary method of choice for the nonambulatory patients. With operative treatment, poor bone quality does not allow early remobilization and functional treatment, and immobilization after surgery is usually inevitable. Postoperative immobilization worsens the muscular situation, such that patients end up having no benefit from surgery. In contrast, ambulatory patients often benefit from orthopedic surgery with ostheosynthesis in order to prevent their losing walking or standing ability. The outcome of orthopedic surgery is much better for ambulatory patients due to better bone stability and preserved mobility. Maintaining the patients' mobility should always be the major aim of an eventual surgical fracture correction.

\section{Scoliosis}

Nearly $100 \%$ of SMA type II patients and type IIIa patients suffer from scoliosis, often with consecutive chest-cage deformities and respiratory involvement. Scoliosis is frequently diagnosed at an early age; patients younger than 4 years of age can suffer from scoliosis, with significant effect on the respiratory system. Predominantly C-shaped thoracolumbar scoliosis can be found, with an average yearly progression in Cobb angle of $5^{\circ}-15^{\circ} .3,29,30$

For patients with SMA type IIIb, preserved standing ability can decelerate the progression of scoliosis, and wellregulated strength training seems to have a slightly positive effect. ${ }^{31}$ Early physiotherapy, as well as walking and standing ability (including passively with technical support), have an important prophylactic relevance with benefit for all patients..$^{30,31}$

Conservative treatment of scoliosis is complicated and ineffective. Corset therapy is rarely indicated in ambulatory patients because of the possible loss of walking ability due to limited trunk mobility. For SMA type II and IIIa, corset therapy does not halt the progression of scoliosis. ${ }^{29-35}$ Nevertheless, it has its value as treatment before possible operative spine stabilization, especially for young children in the growth period, ${ }^{31}$ or if surgery is not possible due to poor general conditions or parental refusal.

Surgical spine correction is the method of choice for nonambulatory patients and is indicated as soon as scoliosis reaches a Cobb angle $>20^{\circ} .{ }^{36}$ Early spinal surgery tends to have better results because of milder scoliosis at the time of surgery and higher flexibility of the spine, such that better correction can be achieved. ${ }^{29,35}$ Furthermore, it improves sitting comfort as well as quality of life, including the patients' selfconfidence due to enhanced appearance. ${ }^{37,38}$ The aim for spine stabilization is to restore sitting ability without arm support and to avoid impingement of the ribs on the pelvis. The best age for intervention is at the earliest 10-12 years, because the patients have reached more than $80 \%$ of their maximal expected height at that time and surgery should be performed as soon as possible for better correction results. ${ }^{30,36}$ Traction treatments as a preparation for the surgery are obsolete. ${ }^{30}$

The three-dimensional correction of scoliosis with alignment of the trunk and head adjustment is more important than anatomical correction. ${ }^{30}$

Formerly performed stabilization with a telescopic rod has shown unsatisfactory results: The idea to provide a height-adaptive instrument was marred by manufacturing deficiency and multiple crankshaft phenomena. ${ }^{36,39}$ An alternative ventro-dorsal access has shown an increased risk for auxiliary abdominal respiratory muscles and the diaphragm, with consecutive reduction of vital capacity. ${ }^{34,40-42}$

The method of choice is the multisegmental dorsal stabilization of the spine from thoracic vertebrae Th2 or Th3 to the sacrum or pelvis, ${ }^{30,36}$ which allows a good segmental correction and stability. Additional advantages include immediate sitting ability postsurgery and early mobilization. ${ }^{36}$ 
Independent of the surgical method, complications such as delayed wound healing and respiratory and neurological complications have been described for SMA patients. ${ }^{30,37,38}$ Furthermore, the anesthetic risk due to reduced respiratory function has to be considered for the surgical and postoperative planning. The relative vital capacity decreases slightly in the postoperative period, but several investigations have shown that it remains largely constant 6-12 months after surgical intervention. ${ }^{30,35}$

For children younger than 10-12 years of age, an adequate technique for spine surgery has not been established. Surgical stabilization performed at a younger age has a high risk for dorsal fusion with crankshaft phenomenon. Some centers have recently performed spine surgery in young children with SMA using growing rods and vertical expandable prosthetic titanium ribs. ${ }^{43-45}$ The effectiveness of these techniques remains to be proven.

\section{Respiratory involvement}

Pulmonary disease remains the major cause of morbidity and mortality in SMA, mainly type I and type II, and respiratory capacity is distinctly reduced at an early age. ${ }^{6}$ Among the 4-6-year-old patients with SMA type II, there is a distinct reduction to an average of $54 \%$ in the relative vital capacity. ${ }^{30}$ For SMA type IIIa, vital capacity is also reduced but with considerably better values. In general, vital capacity decreases progressively with growth but can remain stable after the period of growth far into adult life. ${ }^{30}$ The SMA disease course includes severe weakness of the auxiliary respiratory muscles, mostly sparing the diaphragm, which remains the main respiratory muscle. Muscular imbalance leads to chest-cage deformities that additionally limit the respiratory function. The respiratory muscle weakness results in increased difficulties in clearing respiratory secretions, hypoventilation, recurrent infections, and atelectasis. The recurrent infections exacerbate the muscle weakness, with aggravation of the lung parenchyma. ${ }^{46,47}$

Early physiotherapy with respiratory training is important in order to optimize the remaining auxiliary respiratory muscles and provide expectoration techniques. ${ }^{47,48}$ Preserved standing ability for at least 2 hours per day leads to stabilization of lung function and should be supported with orthoses and standing assistance if necessary. Furthermore, timely treatment of scoliosis allows better ventilation of the lung parenchyma and leads to stabilization of respiratory function. ${ }^{30,35}$

The patient with milder SMA type III, which generally has much less effect on respiratory muscle strength, is at risk for obstructive sleep apnea and exacerbation during acute illness or postoperatively and may develop hypoventilation during adulthood. ${ }^{46}$ Sleep disorders are observed frequently in SMA patients, mostly due to respiratory distress. ${ }^{14,49}$

Lung function, including nocturnal ventilation and coughing ability, should be analyzed frequently and by routine. ${ }^{48}$ Coughing ability is a useful parameter for respiratory muscle function; reduced cough efficiency contributes to pulmonary morbidity and should be improved with mechanical cough assistance. $^{46,48}$

Ventilation support becomes necessary at the time of deterioration of lung function and is recommended for therapeutic SMA management. ${ }^{46-48,50}$ Respiratory support for SMA patients is well established and includes noninvasive ventilation as the method of choice, and invasive ventilation with tracheotomy if necessary. ${ }^{51}$ The long-term aims of ventilation assistance are an improvement of sleep duration and quality, enhancement of functional status, and prolonged survival. Ventilator support should be added at night if sleepdisordered breathing is present. ${ }^{49,52}$ Noninvasive ventilation is often necessary for respiratory failure during viral respiratory infection, recurring pneumonia or atelectasis, and postoperative care, and in the event of severe scoliosis or chest wall deformities. ${ }^{46-48}$

\section{Nutrition problems}

Whereas nutrition is commonly difficult from the beginning in patients with SMA type I, the difficulties are less frequent and constrictive in the other SMA types. Nevertheless, examination is needed in all SMA patients. The primary problems are limited mouth opening with cumulative chewing difficulties and reduced bite force. Increased fatigue of masticatory muscles and swallowing difficulties result in a higher risk for aspiration and consequent pneumonia. A first consensus statement on standard of care in spinal muscular atrophy ${ }^{52}$ recommends examination of silent aspiration in every patient with type I SMA and examination according to clinical status in patients with type II or type III SMA.

Systematic evaluation of weight should be part of routine examination in all SMA patients, ${ }^{53}$ with consideration of the deviating weight gain in these patients due to muscular atrophy and inactivity. Compared with standard weight gain in healthy children, weight gain in SMA patients is mostly in the lower percentiles of weight charts, and poor weight gain can be found in one third of cases. ${ }^{53}$ Also, body mass index significantly underestimates body fat ${ }^{52}$ in SMA patients due to atrophic muscles and abnormal muscle-fat relation; patients are at high risk for obesity despite low body mass index. ${ }^{54-56}$ 
Body composition should therefore be monitored regularly with complementary assessment of nutritional intake. ${ }^{52}$ Nevertheless, since secondary mitochondrial dysfunction with abnormal fatty acid oxidation is documented, ${ }^{52,57,58}$ prolonged fasting should be avoided, especially in cases of acute illness and postoperative care. In case of insufficient nutrition intake due to chewing and swallowing difficulties, a supply with a PEG (percutaneous endoscopic gastrostomy) tube should be considered to prevent malnutrition because weight loss can hardly be balanced after manifestation.

\section{Rehabilitation}

Subsequent to early clinical rehabilitation and mobilization, continued therapy in a rehabilitation care clinic specializing in neuromuscular disorders is recommended. The care center requires both orthopedic and neurological departments to provide multidisciplinary therapy in order to improve the remaining motor capabilities. Starting physiotherapy and respiration training as soon as possible with additional occupational therapy is essential to reintegrate patients into daily activities and to provide education in the use of assisting devices. Daily physiotherapy is important to prevent loss of motoric functions. Active muscle training can optimize the remaining force and condition, but pulmonary restrictions and other activity-limiting factors have to be considered. Because the musculature in SMA patients is less resistant and needs longer regeneration time, it is mandatory to terminate the training before the patient becomes exhausted; training should generally be supervised. Passive movement of the joints is to be included to delay the progression of contractures. Additional training to improve coordination and proprioception and physical procedures like massages, balneotherapeutic measures, heat applications, and electrotherapy can be added. The intensity of the activities should be adapted to the individual constitution of the patient. ${ }^{12,15,31,52}$

\section{Orthoses and technical devices}

As mentioned above, corset therapy cannot halt the progression of scoliosis but remains important to secure the sitting ability during the period before surgery or when patients are not able to undergo spinal surgery due to generally limited condition. ${ }^{59}$ For ambulatory patients, corsets are widely contraindicated because they often restrict walking ability.

For rapidly progressing contractures of upper and lower extremities, night braces can be provided to decelerate contracture progression, so long as they are tolerated by the patient. ${ }^{4,59}$ The positive effect of treatment with night braces is not scientifically proven, however. ${ }^{59}$
Daytime lower-leg orthoses are most commonly indicated for nonambulatory patients with flexible foot deformities, in order to stabilize the feet in sitting position and decelerate the progression of the deformity. Flexible feet deformities are easily corrected in many cases.$^{59}$ Some patients with milder SMA types profit from leg orthoses to steady their gait. ${ }^{4}$ Lower-limb orthoses used postoperatively can improve and maintain the surgical results. For progressive contractures of the lower limbs, surgical release is often indicated. . $^{45}$

Standing position, even when passively maintained through standing aids, is very important for patients with SMA. A prolonged standing ability can reduce scoliosis, decelerate contracture progression, and improve posture and respiratory function. Standing ability should be preserved as long as possible using technical standing and raising devices. ${ }^{15,59}$ Moreover, verticalization is important for the regular psychosocial development of children with SMA. For the passively fixated verticalization, sufficient head control is essential.

The individually designed wheelchair plays a special important role for nonambulatory patients. Patients should be provided with a manual or powered wheelchair by the age of 3 years. ${ }^{4,1531,59-61}$ A powered wheelchair is needed by $60 \%$ of patients with SMA; customized chair design is recommended to improve participation in social life and to optimize posture and sitting assistance. ${ }^{52}$ Cognitive ability is not reduced in SMA patients, ${ }^{62}$ and they are able usually to learn to use and drive a wheelchair, even at an early age. ${ }^{59,61}$

\section{Perioperative management}

SMA patients have an increased risk for postanesthesia complications, which may lead to prolonged intubation, nosocomial infections, tracheotomy, and even death. ${ }^{63}$ Upper-airway obstruction during endotracheal intubation, hypoventilation and atelectasis from impaired cough, and mucociliary clearance due to anesthetic agents are possible perioperative complications that raise the need for extended preoperative evaluation of respiratory status and pulmonary situation. ${ }^{63}$ Preoperative care should include nutritional assessment as well as investigation of gastroesophageal function. ${ }^{64}$ The consensus statement ${ }^{52}$ recommends the measurement of respiratory function and cough effectiveness in addition to physical examination and chest X-ray. Nocturnal respiration and sleep disturbances are additional risk factors; noninvasive ventilation and cough assistance can be indicated prior to surgery, and anesthesia should be performed by an experienced pediatric anesthesiologist because of frequent difficult intubation in almost two thirds of these patients. ${ }^{63}$ However, intraoperative 
anesthesia complications remain very low, and postoperative complications are mostly related to the individual's respiratory status. ${ }^{63}$ Perioperative care should be provided at a multidisciplinary pediatric center with dedicated pediatric anesthesia and postoperative intensive care capacity. ${ }^{52,63}$

\section{Conclusion}

No curative therapy for SMA exists. Nevertheless, several investigations have recently shown that quality of life can be significantly improved and disease progression can be slowed with timely intervention, especially in the orthopedic area. An exact and early diagnosis and classification of SMA, together with an interdisciplinary therapeutic approach, can pave the way to adequate therapy. Essential for affected patients is the provision of wheelchairs and technical devices, if required, and the initiation of prophylactic physiotherapy with respiratory training as soon as possible. Subsequently, patients should be presented to an experienced orthopedic specialist at the soonest in order to provide optimal orthopedic therapy, including adaptive technical devices, and to plan eventual surgical interventions in time for best results. Operative spine stabilization using multisegmental dorsal stabilization is the method of choice for treating scoliosis in nonambulatory patients aged at the earliest 10-12 years. Satisfactory solutions of surgical therapy for severe scoliosis in very young children are lacking.

SMA patients require lifetime medical attendance with an interdisciplinary team for adequate management of orthopedic, respiratory, and nutritional problems. These patients also need social integration and family member education in order to improve their quality of life.

\section{Disclosure}

The authors report no conflicts of interest in this work.

\section{References}

1. Lefebvre S, Bürglen L, Reboullet S, et al. Identification and characterization of a spinal muscular atrophy-determining gene. Cell. 1995;80(1):155-165.

2. Prior TW, Snyder PJ, Rink BD, et al. Newborn and carrier screening for spinal muscular atrophy. Am J Med Genet A. 2010;152A(7): 1608-1616.

3. Granata C, Merlini L, Magni E, Marini ML, Stagni SB. Spinal muscular atrophy: natural history and orthopaedic treatment of scoliosis. Spine (Phila Pa 1976). 1989;14(7):760-762.

4. Evans GA, Drennan JC, Russman BS. Functional classification and orthopaedic management of spinal muscular atrophy. J Bone Joint Surg Br. 1981;63B(4):516-522.

5. Dubowitz V. Chaos in the classification of SMA: a possible resolution. Neuromuscul Disord. 1995;5(1):3-5.

6. D’Amico A, Mercuri E, Tiziano FD, Bertini E. Spinal muscular atrophy. Orphanet J Rare Dis. 2011;6:71.
7. Ignatius J. The natural history of severe spinal muscular atrophy - further evidence for clinical subtypes. Neuromuscul Disord. 1994:4(5-6):527-528.

8. Thomas NH, Dubowitz V. The natural history of type I (severe) spinal muscular atrophy. Neuromuscul Disord. 1994;4(5-6):497-502.

9. MacLeod MJ, Taylor JE, Lunt PW, Mathew CG, Robb SA. Prenatal onset spinal muscular atrophy. Eur J Paediatr Neurol. 1999;3(2):65-72.

10. Felderhoff-Mueser U, Grohmann K, Harder A, et al. Severe spinal muscular atrophy variant associated with congenital bone fractures. J Child Neurol. 2002;17(9):718-721.

11. Zerres K, Rudnik-Schöneborn S, Forrest E, Lusakowska A, Borkowska J, Hausmanowa-Petrusewicz I. A collaborative study on the natural history of childhood and juvenile onset proximal spinal muscular atrophy (type II and III SMA): 569 patients. J Neurol Sci. 1997;146(1):67-72.

12. Zerres K, Rudnik-Schöneborn S. Natural history in proximal spinal muscular atrophy. Clinical analysis of 445 patients and suggestions for a modification of existing classifications. Arch Neurol. 1995;52(5):518-523.

13. Fujak A, Kopschina C, Gras F, Forst R, Forst J. Contractures of the upper extremities in spinal muscular atrophy type II. Descriptive clinical study with retrospective data collection. Ortop Traumatol Rehabil. 2010;12(5):410-419.

14. de Groot IJ, de Witte LP. Physical complaints in ageing persons with spinal muscular atrophy. J Rehabil Med. 2005;37(4):258-262.

15. Fujak A, Wollinsky KH, Forst R. [Proximal spinal muscular atrophy (SMA)]. Z Orthop Unfall. 2007;145(2):233-252. German.

16. Willig TN, Bach JR, Rouffet MJ, Krivickas LS, Maquet C. Correlation of flexion contractures with upper extremity function and pain for spinal muscular atrophy and congenital myopathy patients. Am J Phys Med Rehabil. 1995;74(1):33-38.

17. Fujak A, Kopschina C, Gras F, Forst R, Forst J. Contractures of the lower extremities in spinal muscular atrophy type II. Descriptive clinical study with retrospective data collection. Ortop Traumatol Rehabil. 2011;13(1):27-36.

18. Wang HY, Ju YH, Chen SM, Lo SK, Jong YJ. Joint range of motion limitations in children and young adults with spinal muscular atrophy. Arch Phys Med Rehabil. 2004;85(10):1689-1693.

19. Sporer SM, Smith BG. Hip dislocation in patients with spinal muscular atrophy. J Pediatr Orthop. 2003;23(1):10-14.

20. Zenios M, Sampath J, Cole C, Khan T, Galasko CS. Operative treatment for hip subluxation in spinal muscular atrophy. J Bone Joint Surg Br. 2005;87(11):1541-1544.

21. Granata C, Magni E, Merlini L, Cervellati S. Hip dislocation in spinal muscular atrophy. Chir Organi Mov. 1990;75(2):177-184.

22. Thompson CE, Larsen LJ. Recurrent hip dislocation in intermediate spinal atrophy. J Pediatr Orthop. 1990;10(5):638-641.

23. Shanmugarajan S, Swoboda KJ, Iannaccone ST, Ries WL, Maria BL, Reddy SV. Congenital bone fractures in spinal muscular atrophy: functional role for SMN protein in bone remodeling. J Child Neurol. 2007;22(8):967-973.

24. Fujak A, Kopschina C, Forst R, Gras F, Mueller LA, Forst J. Fractures in proximal spinal muscular atrophy. Arch Orthop Trauma Surg. 2010;130(6):775-780.

25. Vestergaard P, Glerup H, Steffensen BF, Rejnmark L, Rahbek J, Moseklide L. Fracture risk in patients with muscular dystrophy and spinal muscular atrophy. J Rehabil Med. 2001;33(4):150-155.

26. Kinali M, Banks LM, Mercuri E, Manzur AY, Muntoni F. Bone mineral density in a paediatric spinal muscular atrophy population. Neuropediatrics. 2004;35(6):325-328.

27. Khatri IA, Chaudhry US, Seikaly MG, Browne RH, Iannaccone ST. Low bone mineral density in spinal muscular atrophy. J Clin Neuromuscul Dis. 2008;10(1):11-17.

28. Kurihara N, Menaa C, Maeda H, Haile DJ, Reddy SV. Osteoclast-stimulating factor interacts with the spinal muscular atrophy gene product to stimulate osteoclast formation. J Biol Chem. 2001;276(44):41035-41039.

29. Rodillo E, Marini ML, Heckmatt JZ, Dubowitz V. Scoliosis in spinal muscular atrophy: review of 63 cases. J Child Neurol. 1989;4(2): 118-123. 
30. Fujak A, Raab W, Schuh A, Kreß A, Forst R, Forst J. Operative treatment of scoliosis in proximal spinal muscular atrophy: results of 41 patients. Arch Orthop Trauma Surg. 2012;132(12):1697-1706.

31. Fujak A, Forst R, Forst J. [Current strategies of conservative and operative treatment of the most frequent muscular disorders]. Orthopade. 2010;39(1):38-52. German.

32. Brown JC, Zeller JL, Swank SM, Furumasu J, Warath SL. Surgical and functional results of spine fusion in spinal muscular atrophy. Spine (Phila Pa 1976). 1989;14(7):763-770.

33. Phillips DP, Roye DP, Farcy JP, Leet A, Shelton YA. Surgical treatment of scoliosis in a spinal muscular atrophy population. Spine (Phila Pa 1976). 1990;15(9):942-945.

34. Merlini L, Granata C, Bonfiglioli S, Marini ML, Cervellati S, Savini R. Scoliosis in spinal muscular atrophy: natural history and management. Dev Med Child Neurol. 1989;31(4):501-508.

35. Granata C, Cervellati S, Ballestrazzi A, Corbascio M, Merlini L. Spine surgery in spinal muscular atrophy: long-term results. Neuromuscul Disord. 1993;3(3):207-215.

36. Fujak A, Ingenhorst A, Heuser K, Forst R, Forst J. Treatment of scoliosis in intermediate spinal muscular atrophy (SMA type II) in childhood. Ortop Traumatol Rehabil. 2005;7(2):175-179.

37. Modi HN, Suh SW, Hong JY, Cho JW, Park JH, Yang JH. Treatment and complications in flaccid neuromuscular scoliosis (Duchenne muscular dystrophy and spinal muscular atrophy) with posterior-only pedicle screw instrumentation. Eur Spine J. 2010;19(3):384-393.

38. Roso V, Bitu Sde O, Zanoteli E, Beteta JT, de Castro RC, Fernandes AC. [Surgical treatment of scoliosis in spinal muscular atrophy]. Arq Neuropsiquiatr. 2003;61(3A):631-638. Portuguese.

39. Zebala LP, Bridwell KH, Baldus C, et al. Minimum 5-year radiographic results of long scoliosis fusion in juvenile spinal muscular atrophy patients: major curve progression after instrumented fusion. J Pediatr Orthop. 2011;31(5):480-488.

40. Riddick MF, Winter RB, Lutter LD. Spinal deformities in patients with spinal muscle atrophy: a review of 36 patients. Spine (Phila Pa 1976). 1982;7(5):476-483.

41. Shapiro F, Specht L. The diagnosis and orthopaedic treatment of childhood spinal muscular atrophy, peripheral neuropathy, Friedreich ataxia, and arthrogryposis. J Bone Joint Surg Am . 1993;75(11):1699-1714.

42. Miladi LT, Ghanem IB, Draoui MM, Zeller RD, Dubousset JF. Iliosacral screw fixation for pelvic obliquity in neuromuscular scoliosis. A longterm follow-up study. Spine (Phila Pa 1976). 1997;22(15):1722-1729.

43. McElroy MJ, Shaner AC, Crawford TO, et al. Growing rods for scoliosis in spinal muscular atrophy: structural effects, complications, and hospital stays. Spine (Phila Pa 1976). 2011;36(16):1305-1311.

44. Chandran S, McCarthy J, Noonan K, Mann D, Nemeth B, Guiliani T. Early treatment of scoliosis with growing rods in children with severe spinal muscular atrophy: a preliminary report. J Pediatr Orthop. 2011;31(4):450-454.

45. Campbell RM. VEPTR: past experience and the future of VEPTR principles. Eur Spine J. 2013;22 Suppl 2:S106-S117.

46. Schroth MK. Special considerations in the respiratory management of spinal muscular atrophy. Pediatrics. 2009;123 Suppl 4:S245-S249.

47. Manzur AY, Muntoni F, Simonds A. Muscular dystrophy campaign sponsored workshop: recommendation for respiratory care of children with spinal muscular atrophy type II and III. 13th February 2002, London, UK. Neuromuscul Disord. 2003;13(2):184-189.
48. Ioos C, Leclair-Richard D, Mrad S, Barois A, Estournet-Mathiaud B. Respiratory capacity course in patients with infantile spinal muscular atrophy. Chest. 2004;126(3):831-837.

49. Mellies U, Dohna-Schwake C, Stehling F, Voit T. Sleep disordered breathing in spinal muscular atrophy. Neuromuscul Disord. 2004;14(12):797-803.

50. Wallgren-Pettersson C, Bushby K, Mellies U, Simonds A; ENMC. 117th ENMC workshop: ventilatory support in congenital neuromuscular disorders - congenital myopathies, congenital muscular dystrophies, congenital myotonic dystrophy and SMA (II) April 4-6, 2003, Naarden, The Netherlands. Neuromuscul Disord. 2004;14(1):56-69.

51. Wollinsky KH, Mindé A, Schreiber H, Kluger P, Mehrkens HH. [Effectiveness of home ventilation of young children and infants]. Med Klin (Munich). 1995;90(1 Suppl 1):57-59. German.

52. Wang $\mathrm{CH}$, Finkel RS, Bertini ES, et al; Participants of the International Conference on SMA Standard of Care. Consensus statement for standard of care in spinal muscular atrophy. $J$ Child Neurol. 2007;22(8):1027-1049.

53. Messina S, Pane M, De Rose P, et al. Feeding problems and malnutrition in spinal muscular atrophy type II. Neuromuscul Disord. 2008;18(5):389-393.

54. Sproule DM, Montes J, Montgomery M, et al. Increased fat mass and high incidence of overweight despite low body mass index in patients with spinal muscular atrophy. Neuromuscul Disord. 2009;19(6):391-396.

55. Sproule DM, Montes J, Dunaway S, et al. Adiposity is increased among high-functioning, non-ambulatory patients with spinal muscular atrophy. Neuromuscul Disord. 2010;20(7):448-452.

56. Markowitz JA, Singh P, Darras BT. Spinal muscular atrophy: a clinical and research update. Pediatr Neurol. 2012;46(1):1-12.

57. Tein I, Sloane AE, Donner EJ, Lehotay DC, Millington DS, Kelley RI. Fatty acid oxidation abnormalities in childhood-onset spinal muscular atrophy: primary or secondary defect(s)? Pediatr Neurol. 1995;12(1):21-30.

58. Crawford TO, Sladky JT, Hurko O, Besner-Johnston A, Kelley RI. Abnormal fatty acid metabolism in childhood spinal muscular atrophy. Ann Neurol. 1999;45(3):337-343.

59. Fujak A, Kopschina C, Forst R, Mueller LA, Forst J. Use of orthoses and orthopaedic technical devices in proximal spinal muscular atrophy. Results of survey in 194 SMA patients. Disabil Rehabil Assist Technol. 2011;6(4):305-311.

60. Jones MA, McEwen IR, Hansen L. Use of power mobility for a young child with spinal muscular atrophy. Phys Ther. 2003;83(3):253-262.

61. Chung BH, Wong VC, Ip P. Spinal muscular atrophy: survival pattern and functional status. Pediatrics. 2004;114(5):e548-e553.

62. von Gontard A, Zerres K, Backes M, et al. Intelligence and cognitive function in children and adolescents with spinal muscular atrophy. Neuromuscul Disord. 2002;12(2):130-136.

63. Graham RJ, Athiraman U, Laubach AE, Sethna NF. Anesthesia and perioperative medical management of children with spinal muscular atrophy. Paediatr Anaesth. 2009;19(11):1054-1063.

64. Iannaccone ST. Modern management of spinal muscular atrophy. J Child Neurol. 2007;22(8):974-978.
The Application of Clinical Genetics

\section{Publish your work in this journal}

The Application of Clinical Genetics is an international, peer-reviewed open access journal that welcomes laboratory and clinical findings in the field of human genetics. Specific topics include: Population genetics; Functional genetics; Natural history of genetic disease; Management of genetic disease; Mechanisms of genetic disease; Counseling and ethical

\section{Dovepress}

issues; Animal models; Pharmacogenetics; Prenatal diagnosis; Dysmorphology. The manuscript management system is completely online and includes a very quick and fair peer-review system, which is all easy to use. Visit http://www.dovepress.com/testimonials.php to read real quotes from published authors. 